\title{
Optical Afterglows as Probes for the Central Engine and Fireball of Gamma-Ray Bursts $\dagger$
}

\author{
Liang $\mathbf{L i}^{1}$, En-Wei Liang ${ }^{1,2}, \mathrm{He}_{\text {Gao }}{ }^{3}$, and Bing Zhang ${ }^{3,1}$ \\ ${ }^{1}$ Department of Physics and GXU-NAOC Center for Astrophysics and Space Sciences, \\ Guangxi University; lew@gxu.edu.cn \\ ${ }^{2}$ National Astronomical Observatories, CAS, Beijing 100012 \\ ${ }^{3}$ Department of Physics and Astronomy, University of Nevada, Las Vegas, NV 89154
}

\begin{abstract}
Well-sampled optical lightcurves of 146 gamma-ray bursts (GRBs) are compiled from literature. We identify possible emission components based on our empirical fits and present statistical analysis for these components. We find that the flares are related to prompt emission, suggesting that they could have the same origin in different episodes. The shallow decay segment is not correlated with prompt gamma-rays. It likely signals a long-lasting injected wind from GRB central engines. Early after onset peak is closely related with prompt emission. The ambient medium density profile is likely $n \propto r^{-1}$. No correlation between the late re-brightening bump and prompt gamma-rays or the onset bump is found. They may be from another jet component.
\end{abstract}

Keywords. Gamma-ray: bursts, Method: statistics

\section{Introduction}

The most popular model of gamma-ray bursts (GRBs) is the internal + external shock fireball model, which suggests that the prompt emission is produced by internal shocks at a distance internal to the deceleration radius of the GRB fireball and the broadband afterglows are from the external shocks when the fireball is decelerated by the ambient medium. Observations in the Swift era show that the GRB central engines do not die out quickly. The observed afterglow emission is a superposition of the traditional external shock afterglow and an afterglow related to the late central engine activities (e.g., Zhang 2012). We present a comprehensive analysis for the optical emission components based on our empirical fits to the optical lightcurves and constrain on the central engines and the fireball of GRBs with the data. Our sample include 146 GRBs that have a well-sample optical lightcurves. A full version of the sample and our results please refer to Li et al. (2012) and Liang et al. (2012).

\section{Results}

Eight possible components that may have distinct physical origins are identified. We find that the peak luminosity of optical flares are anti-correlated with the peak time (Fig. 1a). Its temporal evolution may signal the evolution of the GRB central engine. The shallow decay component shares a similar relation between the break time and break luminosity with X-rays (Fig. 1b), which may be related to a long-lasting spinning-down

$\dagger$ Supported by the "973" Program of China (2009CB824800), the National Natural Science Foundation of China (Grants No. 11025313), and Special Foundation for Distinguished Expert Program of Guangxi, the Guangxi Natural Science Foundation (2010GXNSFC013011, Contract No. 2011-135), and the 3th Innovation Projet of Guangxi University. BZ acknowledges support from NSF (AST-0908362). 

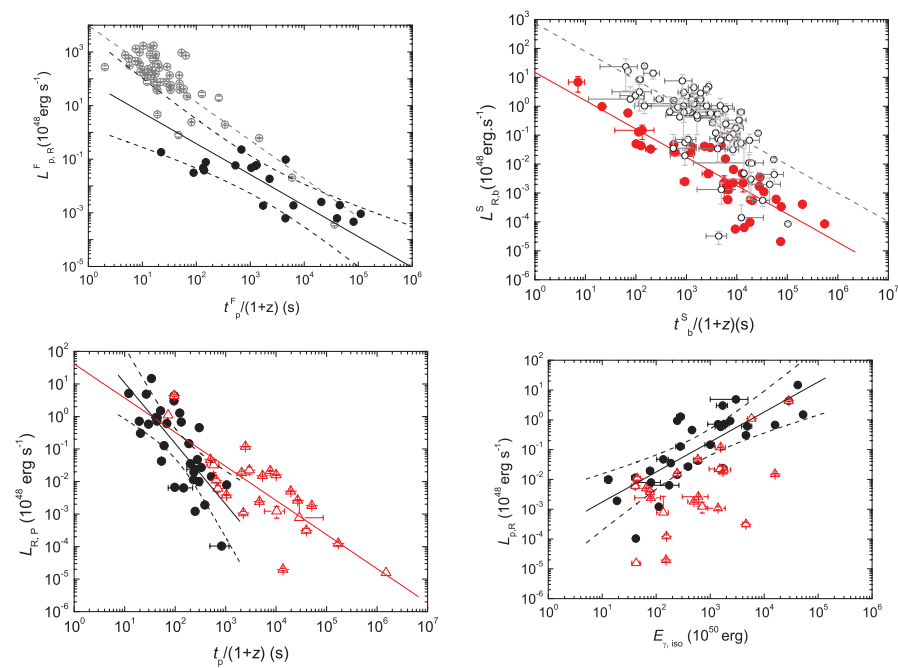

Figure 1. Top panels: The peak luminosity of the optical flares and the luminosity at the break time of the shallow decay segment as a function of the corresponding times. The X-ray data (grey dots) are also shown for comparison. Low panels: peak luminosity of the onset (solid circles) and re-brightening (opened triangles) bumps as a function of the peak times and as a function of $E_{\gamma, \text { iso }}$. Lines are the best fits and the dashed curves mark the $3 \sigma$ significance level of the fits.

central engine or piling up of flare materials onto the blast wave. The rising and decaying slopes for both the onset and re-brightening bumps are in the range of $0.3 \sim 4$ (typically $\sim 1.5$ ) and in $0.6 \sim 1.8$ (typically $\sim 1.15$ ), respectively. The peak luminosity is anticorrelated with the peak time, with the power-law indices $-1.86 \pm 0.29$ for the onset bumps and $-1.05 \pm 0.16$ for the re-brightening bumps (Fig. 1c). The peak luminosity and the isotropic energy release of the onset bumps are correlated with $E_{\gamma, \text { iso }}$, whereas no similar correlation is found for the re-brightening bumps (Fig. 1d). These results suggest that the afterglow onset bumps are likely due to the deceleration of the GRB fireballs. They are good probes for the properties of the fireballs and their ambient medium density profile. Within the framework of the synchrotron external shock model, we obtain the power-law index of the relativistic electrons in the range of 2 to 3.5 among bursts (typically 2.5), and the medium density profile as $n \propto r^{-1}$, an intermediate profile between the constant-density inter-stellar medium and a stellar wind. With the medium density profile obtained from our analysis, we also confirm the $\Gamma_{0}-E_{\text {iso }, \gamma}$ relation in our previous work. The lack of correlations between the re-brightening bump properties with the prompt emission properties imply that they may originate from an independent jet component. We find that this jet component is likely on-axis and is independent of the prompt emission jet component. This component may have a kinetic energy budget comparable to the prompt emission component, but with a lower initial Lorentz factor, typically several tens.

\section{References}

Li, L., Liang, W., Tang, Q.-W. et al. 2012, ApJ, 758, 27

Liang, W., Li, L., He G. et al. 2012, ApJ, submitted

Zhang, B. 2012, IAU Symposium, 279, 102 